\title{
miR-144-3p regulates the resistance of lung cancer to cisplatin by targeting Nrf2
}

\author{
YAN YIN $^{1 *}$, HUA LIU $^{2-5^{*}}$, JUNHUI XU $^{1}$, DONGSHENG SHI ${ }^{1}$, LIANG ZHAI $^{1}$, \\ BIN LIU $^{1}$, LEI WANG ${ }^{1}$, GUANGXIN LIU ${ }^{1}$ and JIANWEN QIN ${ }^{1}$ \\ ${ }^{1}$ Respiratory and Critical Care Medicine of Tianjin Chest Hospital, Tianjin 300222; \\ ${ }^{2}$ Department of Hepatology and Gastroenterology Tianjin Third Central Hospital of Tianjin, \\ Tianjin 300170; ${ }^{3}$ Tianjin Institute of Hepatobiliary Disease; ${ }^{4}$ Tianjin Key Laboratory of Artificial Cells; \\ ${ }^{5}$ Artificial Cell Engineering Technology Research Center of Public Health Ministry, Tianjin 300170, P.R. China
}

Received May 21, 2018; Accepted August 24, 2018

DOI: $10.3892 /$ or.2018.6772

\begin{abstract}
Chemotherapeutic drug resistance is correlated with treatment failure and poor prognosis among lung cancer patients. Numerous studies indicate the relevance of miRNAs in inducing certain drug resistance. In the course of the study, we unexpectedly found that miR-144-3p could regulate the cisplatin resistance of lung cancer cells via Nrf2. However, Nrf2 also could reverse activate the expression of miR-144-3p by binding to the ARE box in the miR-144-3p promoter. This may be a self-protection mechanism of the body. In addition, we also found that in other cancer cell lines, such as HepG2, miR-144-3p also had the function of regulating cisplatin resistance. These findings may provide some theoretical reference for the clinical inhibition of cisplatin resistance.
\end{abstract}

\section{Introduction}

Lung cancer is a malignant tumor with the highest incidence and mortality in the world $(1,2)$. The 5-year survival rate has not improved significantly in the past 25 years, and is approximately $18 \%$ (3), even with specialized treatment combination of surgery, chemotherapy, and radiation therapy (4). For lung cancer patients, one of the most important causes for such a low survival rate is the occurrence of drug resistance that develops during the chemotherapeutic procedure (5). Therefore, in order to obtain better results in lung cancer therapy, it is crucial to find effective ways to counter the drug resistance by exploring the underlying mechanisms of chemoresistance. Cisplatin is

Correspondence to: Professor Jianwen Qin, Respiratory and Critical Care Medicine of Tianjin Chest Hospital, Tianjin 300222, P.R. China

E-mail: qinjianwen34@sohu.com

${ }^{*}$ Contributed equally

Key words: miR-144-3p, Nrf2, cisplatin-resistance, lung cancer widely used in the clinical treatment of lung cancer due to its marked anticancer effect, and broad anticancer spectrum (6), and its main function is through inhibition of DNA synthesis to induce tumor cell apoptosis $(7,8)$. Resistance to cisplatin seriously affects the clinical efficacy of lung cancer, and its main mechanism has not been elucidated clearly. Recently, the effects of miRNAs on the development of cancer drug resistance has gained attention.

MicroRNAs (miRNAs) are small non-coding RNA molecules (approximately 20-25 nucleotides) which are known to negatively modulate the expression of targeted genes by completely or partially binding with the 3'-untranslated region (3'-UTR) of the mRNA (9-11). This function of miRNAs plays an important role in the development of various malignancies $(12,13)$. Aberrant miRNA expression has been observed in both physiological and pathological processes, such as apoptosis and chemotherapy resistance, in multiple human cancers (14).

Nuclear factor erythroid 2-like 2 (NFE2L2; commonly known as Nrf2) is a critical transcription factor in the regulation of antioxidant and cytoprotective genes, by binding to and activating the expression of promoters containing the antioxidant response element (ARE). Nrf2 has been reported to be targeted by many miRNAs. miR-27a-5p, miR-142-5p, miR-28 and miR-93-5p suppressed cancer chemoprevention activity by downregulating the Nrf 2 mRNA and protein levels (15). miR-93 was able to regulate the oncogenic process in mammaries through regulation of its target gene NRF2 (16). MicroRNA-140-5p aggravated doxorubicin-induced cardiotoxicity by promoting myocardial oxidative stress by targeting Nrf2 and Sirt2 (17). Nevertheless, the biological role of miR-144-3p in modulating lung cancer drug resistance by targeting Nrf2 is not well understood.

In the present study, we investigated the expression levels of miR-144-3p in tumor and adjacent tissues of lung cancer patients and in lung cancer cell lines, in order to identify the functional role of miR-144-3p in lung cancer biology. In addition, we elucidated the regulatory cisplatin resistance involving miR-144-3p and Nrf2 in lung cancer cell multidrug resistance. Furthermore, in the course of the experiment, we unexpectedly found that there was a regulation loop between 
miR-144-3p and Nrf2 in regulating the cisplatin resistance of lung cancer cells.

\section{Materials and methods}

Cell culture. A549, H1299, HepG2, MCF-7, HeLa and Cos-7 cell lines were used in the present study. The A549 and H1299 cell lines were purchased from the American Type Culture Collection (ATCC; Manassas, VA, USA) (nos. CCL-185 and CRL-5803, respectively). These cells were seeded in RPMI-1640 medium and fetal bovine serum (FBS) to a final concentration of $10 \%$. HepG2, MCF-7, HeLa and Cos-7 cells used in this study were purchased from ATCC (nos. HB-8065, HTB-22, CCL-2 and CRL-1651, respectively). These cells were seeded in Dulbecco's modified Eagle's medium-high glucose (DMEM-HG; Hyclone; GE Healthcare Life Sciences, Logan, UT, USA) supplemented with 10\% FBS) (Gibco; Thermo Fisher Scientific, Inc., Waltham, MA, USA). All of the aforementioned cell lines were cultured at $37^{\circ} \mathrm{C}$ in a humidified air with $5 \% \mathrm{CO}_{2}$.

Plasmid constructs and cell transfection. Human Nrf2 (Gene ID: 4780) was inserted into expression vector (pcDNA3.1-). ShNrf2 and its corresponding control were kindly provided by Professor Jian Dong (North Carolina State University, Raleigh, NC, USA). miR-144-3p mimic, miR-144-3p inhibitor or the appropriate negative controls (NC) of miRNA mimic (mimic $\mathrm{NC}$ ) and miRNA inhibitor (inhibitor NC) were obtained from Guanzhou RiboBio Co., Ltd. (Guanzhou, China). For transfection experiments, the cells were cultured in growth medium without antibiotics at $60 \%$ confluence for 2 days, and then transfected with transfection reagent (FuGENE ${ }^{\circledR}$ HD; Roche Diagnostics, Basel, Switzerland) according to the manufacturer's instructions. After incubation for $6 \mathrm{~h}$, the medium was removed and replaced with normal culture medium for $48 \mathrm{~h}$.

MTT assay. Cell growth was estimated by a modified MTT assay. As a measurement of cell growth, the cells were seeded onto a 96-well dish and grown in medium containing $10 \%$ FBS. After the cells were treated daily with cisplatin $(1,2,4$, $8,16,32$ and $64 \mu \mathrm{M})$ for $24 \mathrm{~h}$, the MTT reagent $(2.5 \mathrm{mg} / \mathrm{ml})$ was added and the optical density $(570 \mathrm{~nm})$ was measured by ELISA reader.

Quantitative real-time RT-PCR. Total RNA, including miRNA, was extracted by using a e.Z.N.A miRNA kit (Omega Bio-Tek, Inc., Norcross, GA, USA) according to the manufacturer's protocol, and the sample was reverse-transcribed using M-MLV reverse transcriptase (Promega Corporation, Madison, WI, USA). Real-time PCR was performed using Applied Biosystems Step One ${ }^{\mathrm{TM}}$ Real-Time PCR System. Fast SYBR ${ }^{\circledR}$ Green Master Mix was obtained from Applied Biosystems; Thermo Fisher Scientific, Inc. Data presented as the relative expression levels of Nrf2, HO-1, NQO-1, Bcl-2 and caspase- 3 were normalized by GAPDH. The primers for the PCR analysis are listed in Table I. Amplification of U6 small nuclear RNA served as an endogenous control used to normalize miR-144-3p expression data. Thermocycling conditions were as follows: $95^{\circ} \mathrm{C}$ for $5 \mathrm{~min}$ followed by 40 cycles at $95^{\circ} \mathrm{C}$ for $10 \mathrm{sec}$ and $60^{\circ} \mathrm{C}$ for $30 \mathrm{sec}$, then a melting curve analysis from 60 to $95^{\circ} \mathrm{C}$ every $0.2^{\circ} \mathrm{C}$ for $1.5 \mathrm{~min}$ was obtained. Each sample was analyzed in triplicate, and quantified using the $2^{-\Delta \Delta \mathrm{Cq}}$ method (18).

Protein extraction and western blotting. For the western blot analysis, protein samples were extracted from the cells with Protein Extraction Reagent (Pierce; Thermo Fisher Scientific, Inc.). The concentrations of the proteins were determined using the BCA Quantification kit (Beyotime Institute of Biotechnology, Beijing, China) for subsequent sodium dodecyl sulfate-polyacrylamide gel electrophoresis (SDS-PAGE). The proteins $(20 \mu \mathrm{g})$ were separated by SDS PAGE $(10 \%)$ and transferred onto a PVDF membrane. The membrane was blocked using 5\% non-fat milk at $25^{\circ} \mathrm{C}$ for $1 \mathrm{~h}$, and then incubated with primary antibodies overnight at $4^{\circ} \mathrm{C}$. The antibodies used were as follows: Anti-human GAPDH antibody (dilution 1:2,000; cat. no. 97166; Cell Signaling Technology, Inc., Danvers, MA, USA), anti-human Nrf2 antibody (dilution 1:1,000; cat. no. sc-81342; Santa Cruz Biotechnology, Inc., Dallas, TX, USA), anti-human HO-1 antibody (dilution 1:1,000; cat. no. 5853; Cell Signaling Technology, Inc.), anti-human NQO-1 antibody (dilution 1:1,000; cat. no. ab28947; Abcam, Cambridge, UK), anti-human Bcl-2 antibody (dilution 1:1,000; cat. no. sc-509) and anti-human caspase-3 antibody (dilution 1:1,000; cat. no. sc-271759) (both from Santa Cruz Biotechnology, Inc.). Then, the membrane was incubated with IRDyeTM-800 conjugated anti-mouse or anti-rabbit secondary antibodies (dilution 1:5,000; cat. nos. 115-005-146 and 115-005-144; Jackson ImmunoResearch Laboratories, Inc., West Grove, PA, USA) for $1 \mathrm{~h}$ at room temperature. The protein signals were visualized with the Odyssey Infrared Imaging System (LI-COR Biosciences, Lincoln, NE, USA). GAPDH expression was used as an internal control.

Luciferase constructs, site-mutation, and luciferase assay. The luciferase constructs were as follows: i) Nrf2-3'-UTR-WT: The Nrf2 3'-UTR region was fused to the pmirGLO reporter vector; ii) Nrf2-3'-UTR-MUT: The same as the Nrf2-3'-UTR-WT, except that the miR-144-3p binding site was mutated; iii) miR-144-3p-luc: The miR-144-3p promoter region (about $2000 \mathrm{bp}$ ) was fused to the pGL3-Basic reporter vector; iv) Mut-miR-144-3p-luc: The same as miR-144-3p-luc, except that the Nrf2 binding site was mutated. The cells ( $2 \times 10^{5} /$ well) were plated in 24 -well plates. To ascertain the effect of miR-144-3p on Nrf2, Cos-7 cells were co-transfected with miR-144-3p mimic or mimic NC in combination with Nrf2-3'-UTR-WT or Nrf2-3'-UTR-MUT. In order to explore the mechanism of Nrf2 acting on miR-144-3p, Cos-7 cells were co-transfected with Nrf2 or control (pcDNA3.1-) in combination with miR-144-3p-luc, or mut-miR-144-3p-luc. Cells were harvested $48 \mathrm{~h}$ after transfection and luciferase activity was assessed using the Dual Luciferase Assay System (Promega Corp.). The results were expressed as a fold induction relative to the cells transfected with the control after normalization to Renilla activity. In the dual luciferase assay results, all columns represented the mean result of three independent experiments and the error bars represented the standard deviation.

Chromatin immunoprecipitation (ChIP) assay. We used a commercial Chromatin Immunoprecipitation (ChIP) Assay kit 
Table I. Primers used for RT-PCR.

\begin{tabular}{ll}
\hline Nrf2 & F: TCAGCGACGGAAAGAGTA \\
& R: GGGAGTAGTTGGCAGATC \\
FO-1 & F: GTGAAGCGGCTCCACGAG \\
& R: GGCAATGTTGGGGAAGGT \\
Faspase-3 & F: GAGTTCGGTGGGGTCATG \\
& R: GGAGAAATCAAACAGAGGC \\
Bcl-2 & R: GAGTTCGGTGGGGTCATG \\
& F: GAAAGGACATCACAGGTAA \\
NQO-1 & R: GGGAACTGGAATATCACAA \\
& F: CTCCTCCACCTTTGACGC \\
GAPDH & R: CCACCACCCTGTTGCTGT
\end{tabular}

F, forward; R, reverse.

(Merck KGaA, Darmstadt, Germany) by following the manufacturer's instructions. After the treatment, each test group was incubated with $1 \%$ formaldehyde to cross-link DNA-protein complexes. After washing with ice-cold PBS three times, the cells were lysed in SDS lysis buffer. Then lysates were sonicated to shear DNA to $200-1,000$ bp fragments. We then used an anti-Nrf2 antibody to immunoprecipitate the cross-linked protein at $4^{\circ} \mathrm{C}$ overnight. IgG acted as the negative control. The DNA was used as a template for PCR and utilized the Nrf2 binding site. The PCR products were separated on $1 \%$ agarose gel.

Statistical analysis. High throughput sequencing data and prognostic data were derived from the TCGA database (GSE56036) and LinkedOmics database (ID-3650). The software of TargetScan was used to predict gene targets. Data were expressed as the mean $\pm \mathrm{SE}$, accompanied by the number of experiments performed independently, and analyzed by t-tests. Differences at $\mathrm{P}<0.05$ were considered to be statistically significant.

\section{Results}

miR-144-3p expression is downregulated in lung cancer tissues and associated with poor prognosis. There was a significant difference of miR-144-3p expression in tumor tissues and normal tissues in patients with platinum insensitivity drugs by analyzing high-throughput sequencing data from the TCGA database (GSE56036). The results revealed that miR-144-3p expression was significantly decreased in lung cancer tissues compared with adjacent non-tumor tissues (Fig. 1A-C). The emergence of drug resistance often indicates a poor prognosis. To assess the clinical significance of miR-144-3p overexpression in lung cancer, we evaluated the association between miR-144-3p levels and patient clinicopathological characteristics based on the TCGA database (GSE56036) and LinkedOmics database (ID-3650). Kaplan-Meier survival analysis revealed that patients with higher miR-144-3p levels had longer overall survival and progression-free survival times than those who had lower levels of miR-144-3p (Fig. 1D). These findings indicated that the expression of miR-144-3p was decreased in lung cancer tissues and associated with poor prognosis. Thus, we performed experimental research on whether miR-144-3p was related to cisplatin resistance.

miR-144-3p inhibits cisplatin resistance in lung cancer cells. To further investigate whether miR-144-3p was involved in drug resistance in lung cancer, we transfected miR-144-3p mimic or miR-144-3p inhibitor to lung cancer cells (Fig. 2A and B) and then exposed the cells to cisplatin with rising levels of concentration for $24 \mathrm{~h}$. The viability of lung cancer cells that were transfected with miR-144-3p were more sensitive to cisplatin in comparison with the mimic NC group (Fig. 2C and D). However, the miR-144-3p inhibitor did the opposite, the resistance of cells was significantly enhanced to cisplatin (Fig. 2C and D). These results indicated that miR-144-3p inhibited cisplatin resistance in lung cancer cells. Thus, targeting miR-144-3p could reverse the cisplatin resistant behavior in lung tumor cells.

miR-144-3p inhibits the Nrf2 pathway during the cisplatin resistance process in lung cancer cells. In order to further explore how miR-144-3p regulated drug resistance, we used TargetScan to predict potential miR-144-3p target genes. Nrf2 was identified during the scanning process. Nrf2, as a nuclear transcription factor, which binds to the ARE sequences and activates the molecules downstream, was considered to render cancer cells resistant to drugs including cisplatin. We next investigated whether miR-144-3p could regulate the Nrf2 pathway during the cisplatin resistance process in lung cancer cells.

Within 24 h of cisplatin treatment, we examined the mRNA and protein levels of Nrf2 in lung cancer cells after transfection with the miR-144-3p mimic or miR-144-3p inhibitor. Data revealed that overexpressed miR-144-3p effectively suppressed the mRNA and protein levels of Nrf2 in lung cancer cells (Fig. 3A and B). However, the mRNA and protein levels of Nrf2 were upregulated in the miR-144-3p inhibitor-transfected lung cancer cells (Fig. 3C and D). Concurrently, the expression of Nrf2 downstream target genes, which are involved in drug resistance, were examined. As revealed in Fig. 3E-H, the expression of HO-1, NQO1 and Bcl-2 was downregulated significantly in the miR-144-3p mimic-transfected group compared to the miR-144-3p mimic-NC group. However, the miR-144-3p inhibitor-transfected group exhibited the opposite results. Then, we investigated the expression of caspase-3 by Real-time PCR and western blot assays. The results confirmed that the expression of caspase-3 and miR-144-3p exhibited a positive association trend (Fig. 3E-H). These results revealed that miR-144-3p could inhibit the expression of Nrf2 against the drug resistance of lung cancer cells.

miR-144-3p promotes cisplatin sensitivity by adjusting Nrf2 in lung cancer cells. To further determine that the effect of miR-144-3p in cisplatin resistance was achieved by regulating Nrf2, we generated two stable lung cancer cell lines with Nrf2 knocked down (shNrf2 group). Then miR-144-3p was overexpressed or inhibited in the shNrf2 stable lung cancer cells. We used Real-time PCR to assess the mRNA levels of HO-1, NQO1, Bcl-2 and caspase-3. Concurrently, western blotting was also used to detect the protein levels of HO-1, NQO1, Bcl-2 and 
A

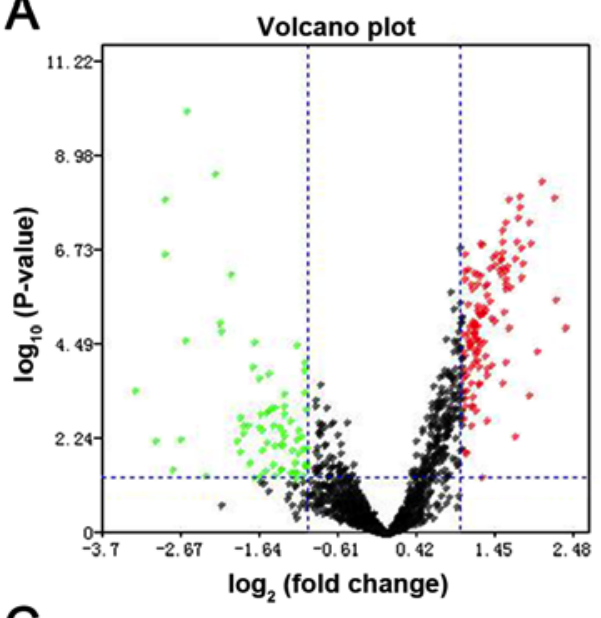

C

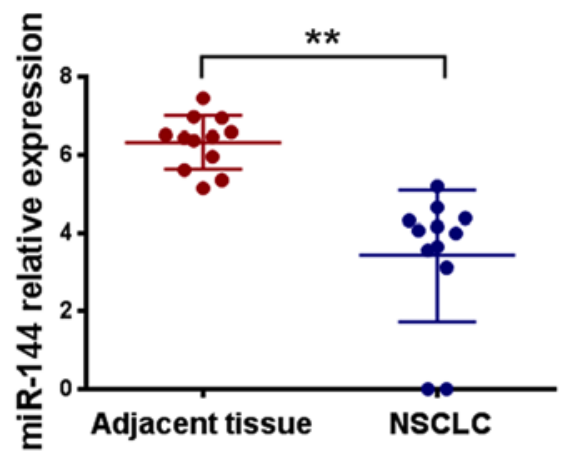

B

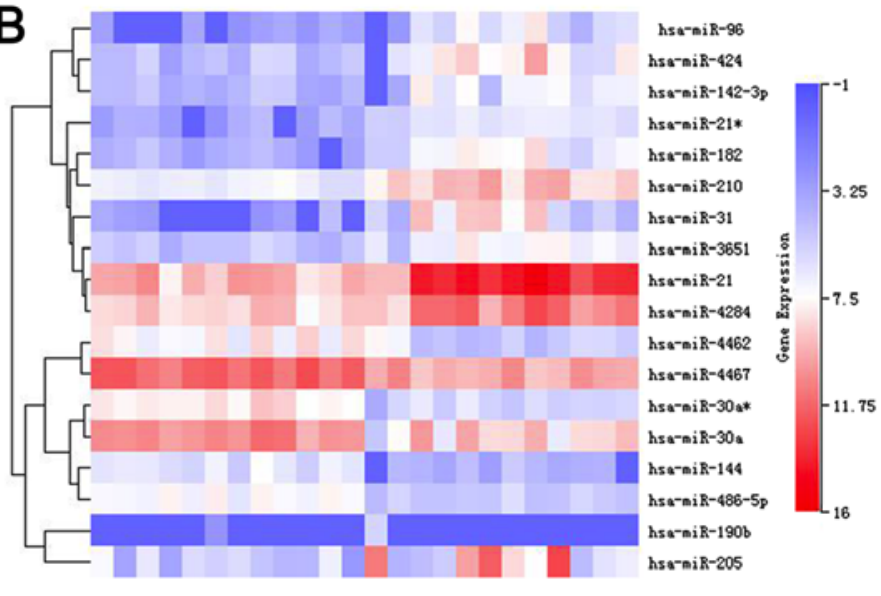

D

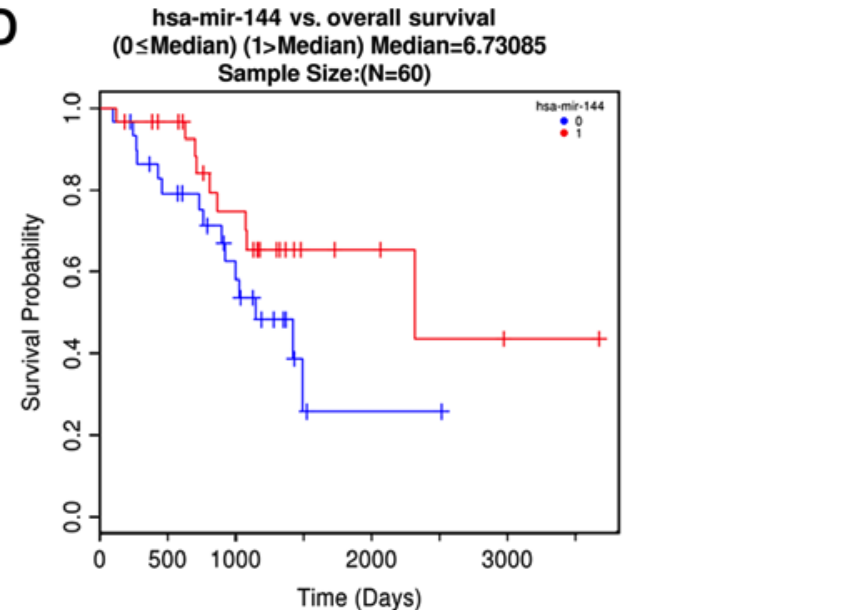

Figure 1. miR-144-3p expression is downregulated in lung cancer tissues and associated with poor prognosis. (A-C) Relative expression of miR-144-3p in lung cancer tissues compared with normal tissue was analyzed using TCGA data ( $\left.{ }^{* *} \mathrm{P}<0.01\right)$. (D) Kaplan-Meier overall survival curves according to miR-144-3p expression levels. Error bars indicate the mean \pm standard errors of the mean.

caspase-3. The results confirmed that, when Nrf2 was knocked down, miR-144-3p lost the ability to regulate the resistance in cisplatin-treated lung cancer cells (Fig. 4A and B). All of the aforementioned results indicated that miR-144-3p required Nrf2 to promote cisplatin sensitivity in lung cancer cells.

miR-144-3p mediates Nrf2 expression by targeting the 3'-UTR in lung cancer cells. As reported in research, miRNAs are known to negatively modulate the expression of targeted genes by completely or partially binding with the 3'-untranslated regions (3'-UTR). Thus, we constructed two plasmids of luciferase reporter gene vectors named Nrf2-3'-UTR-WT and Nrf2-3'-UTR-MUT respectively. We then examined the effect of miR-144-3p on the Nrf2 3'-UTR by luciferase assay. The results confirmed that miR-144-3p could bind to the 3'-UTR region of Nrf2 directly, reducing the mRNA level of Nrf2 (Fig. 5A and B). The experimental results revealed that $\mathrm{miR}-144-3 \mathrm{p}$ mediated $\mathrm{Nrf} 2$ expression by targeting the 3 '-UTR in lung cancer cells.

Nrf2 also affects the expression of miR-144-3p by binding to the ARE box in the miR-144-3p promoter. Previous research confirmed that miR-144-3p could inhibit the cisplatin resistance of lung cancer cells via Nrf2, and we revealed the possible molecular mechanisms between miR-144-3p and
Nrf2 in this process. Notably, we found that Nrf2 could reverse regulate the expression of miR-144-3p. There was one potential ARE box on the miR-144-3p promoter region. As revealed in Fig. 6A and B, the results of Real-time PCR indicated that the mRNA level of miR-144-3p was positively associated with the mRNA level of Nrf2, whether Nrf2 was overexpressed or knocked down. Nrf2 regulated target genes by binding to the ARE box existing in the promoter region. There was one potential ARE box on the miR-144-3p promoter region. The results of the luciferase assay indicated that the transcriptional activity of miR-144-3p promoter could be upregulated by $\mathrm{Nrf} 2$. However, the miR-144-3p transcriptional activity was not affected when the ARE box was mutated (Fig. 6C). These results may indicate that Nrf2 affected the miR-144-3p transcriptional activity by binding to the ARE box. To further assess the mechanism of this regulation, we used chromatin immunoprecipitation (ChIP) to investigate in lung cancer cells. Data revealed that Nrf2 could be combined with the ARE box, consistent with results of the luciferase assay (Fig. 6D).

miR-144-3p also regulates cisplatin resistance in other tumor cells. In order to clarify whether miR-144-3p played a crucial role to cisplatin resistance in other tumor cells, we exposed HepG2, HeLa, MCF-7 to cisplatin with rising levels of concentration for $24 \mathrm{~h}$ after transfection miR-144-3p 

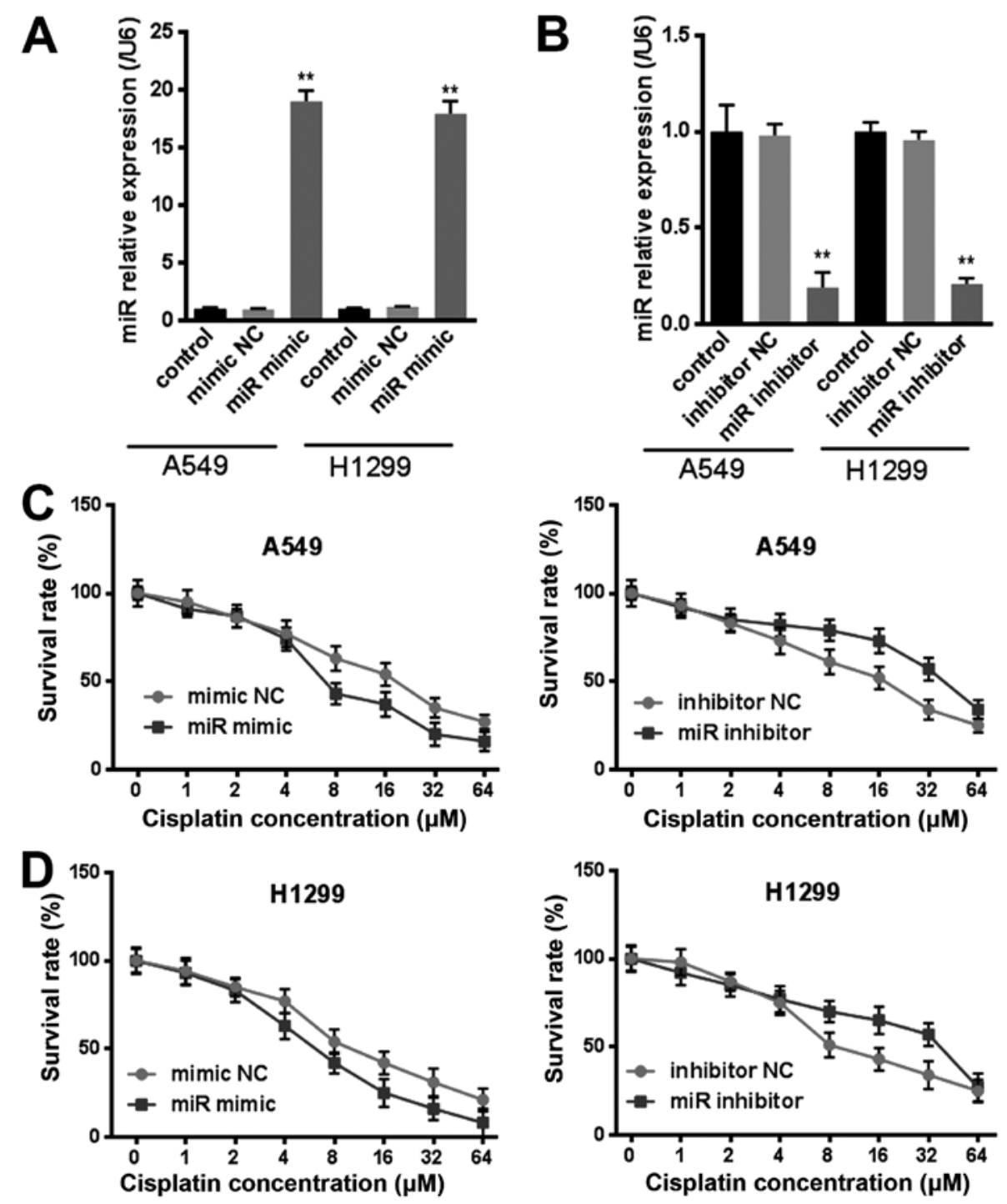

Figure 2. miR-144-3p inhibits cisplatin resistance in lung cancer cells. (A and B) A549 and H1299 cells were transfected with miR-144-3p mimic or inhibitor and their corresponding control mimic NC or inhibitor NC for $48 \mathrm{~h}$ and the miR-144-3p level was detected by Real-time PCR (n $\geq 3$; $\left.{ }^{* *} \mathrm{P}<0.01\right)$. (C and D) A549 and H1299 cells of overexpressing or knocked down miR-144-3p were treated with increasing concentrations $(1,2,4,8,16,32$ and $64 \mu \mathrm{M})$ of cisplatin for $24 \mathrm{~h}$. Cell proliferation was determined by MTT assay. Data (mean \pm SD) represent the mean value of three independent experiments.

mimic or miR-144-3p inhibitor (Fig. 7A-C). The viability of these tumor cells that were transfected with miR-144-3p were more sensitive to cisplatin in comparison with the mimic NC group. Conversely, the resistance of cells after transfection the miR-144-3p inhibitor was significantly enhanced to cisplatin. These results indicated that miR-144-3p could also regulate cisplatin resistance in other tumor cells.

\section{Discussion}

Chemotherapy is one of the main treatments for malignant tumors. However, congenital or acquired resistance, especially multidrug resistance, often leads to chemotherapy failure which has become an unavoidable problem in the process of cancer treatment in clinics. However, there have been limited effective solutions to solve the resistance problem in cancer treatment over the years (19-21). Searching for new chemical resistance mechanisms as well as new molecular targets are urgent in order to solve drug resistance.
In recent years, research has revealed that the aberrant expression of microRNAs (miRNAs) is closely related to the occurrence, development, prognosis and drug resistance of cancer. Although its underlying mechanism still remains uncertain, current experiments and observations have revealed that miRNAs are involved in multidrug resistance processes and play a crucial role in a variety of signaling pathways through negative regulation. For instance, miR-181a, miR-302a and miR-487a can increase the sensitivity of breast cancer to mitoxantrone by inhibiting BCP expression (22-24). In addition, microRNA-130b targets PTEN to induce resistance to cisplatin in lung cancer cells by activating the Wnt/ $\beta$-catenin pathway (25). These discoveries suggest that miRNAs may be used as a target for predicting chemosensitivity and reversing resistance to tumor therapy. In the present study, we determined that the expression of miR-144-3p was significantly downregulated in lung cancer tissue compared to matched adjacent normal lung tissue and associated with poor prognosis, especially in advanced patients with lung cancer. 
A

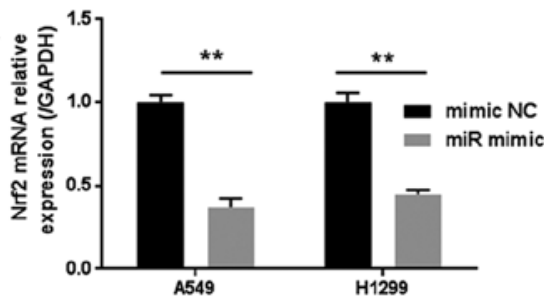

C

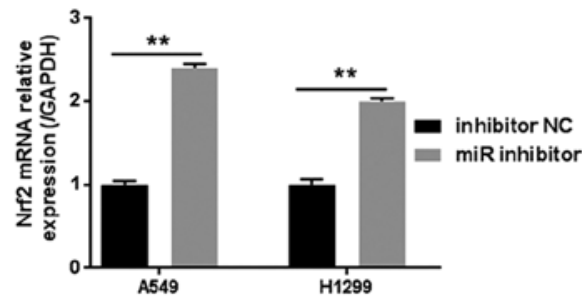

B
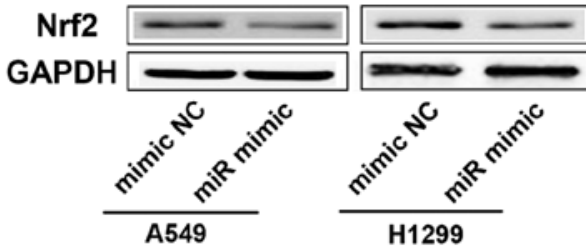

D

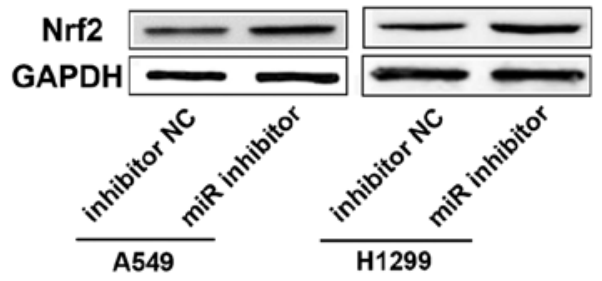

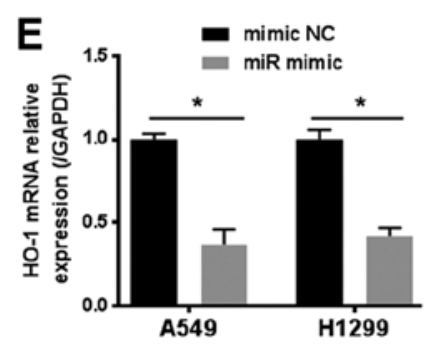
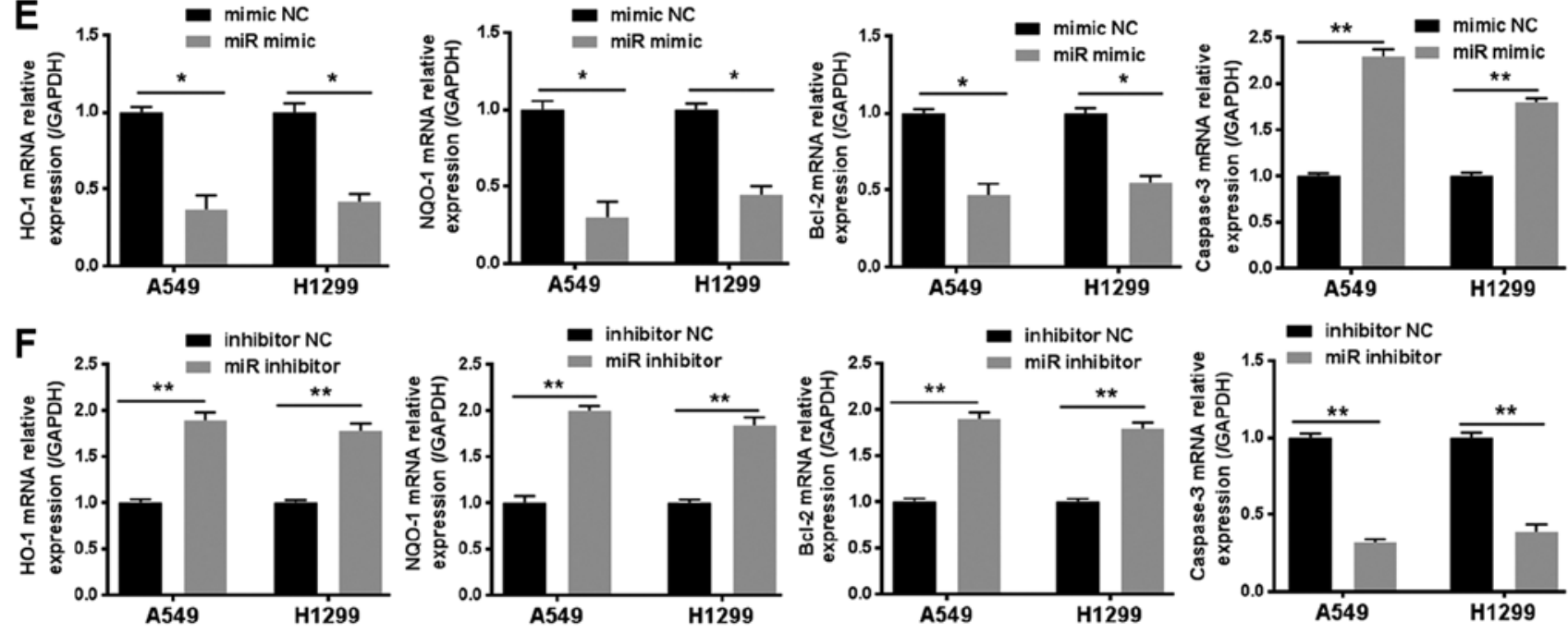

G
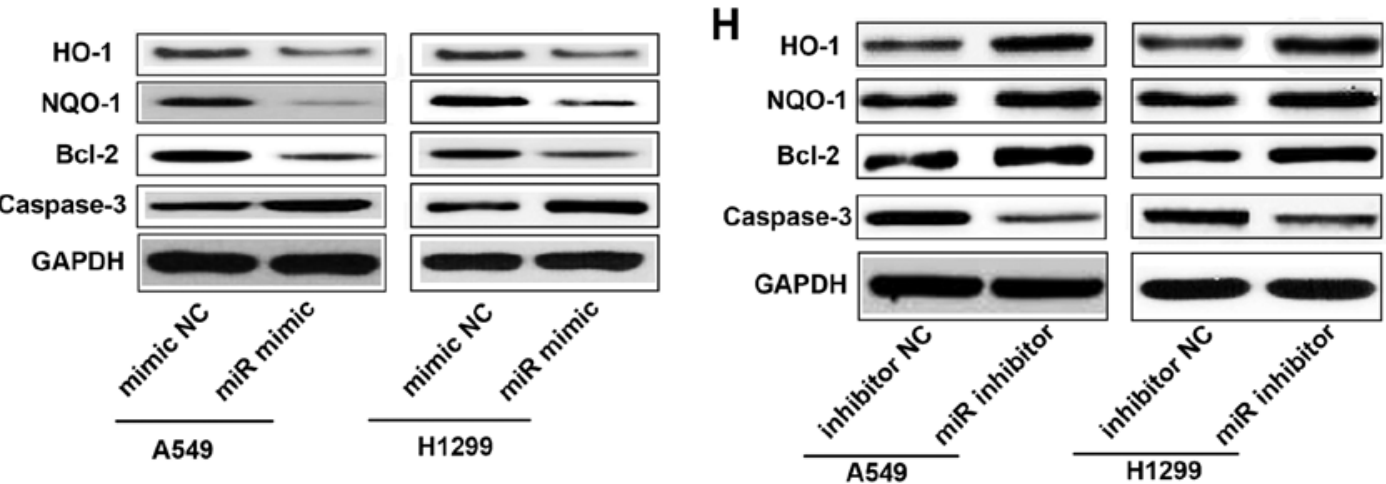

Figure 3. miR-144-3p inhibits the Nrf2 pathway during the cisplatin resistance process in lung cancer cells. (A and C) After treatment with $40 \mu \mathrm{M}$ cisplatin for $24 \mathrm{~h}$, the Nrf 2 mRNA levels (relative to those of GAPDH) in A549 and H1299 cells of overexpressing or knocked down miR-144-3p were individually determined by Real-time PCR ( $\left.\geq 3 ;{ }^{* *} \mathrm{P}<0.01\right)$. (B and D) Western blotting assays were performed to quantitatively assess the protein levels of Nrf2. GAPDH expression was used as an internal control to show equal loading of the protein samples. (E-H) Within $24 \mathrm{~h}$ of $40 \mu \mathrm{M}$ of cisplatin treatment, we examined the mRNA and protein levels of target genes in the downstream of Nrf2 in A549 and H1299 cells after transfection with miR-144-3p mimic or miR-144-3p inhibitor $\left(\mathrm{n} \geq 3 ;{ }^{*} \mathrm{P}<0.05,{ }^{* *} \mathrm{P}<0.01\right)$.

Notably, we found that the change of miR-144-3p levels was related to cisplatin resistance. To further demonstrate whether miR-144-3p was involved in drug resistance in lung cancer, we transfected miR-144-3p mimic or miR-144-3p inhibitor to lung cancer cells and then exposed these cells to cisplatin with increasing levels of concentration. The viability of lung cancer cells that were transfected with miR-144-3p were more sensitive to cisplatin in comparison with the mimic NC group, while with the miR-144-3p inhibitor the opposite was observed.
These findings indicated that miR-144-3p inhibited cisplatin resistance in lung cancer cells. Furthermore, miR-144-3p played a crucial role in cisplatin resistance in other tumor cells, such as HepG2, HeLa and MCF-7.

Nrf2 is an important molecule associated with the resistance of anticancer drugs and activates the molecules downstream, such as antioxidant molecules, detoxification proteins and inhibits cell apoptosis (Bcl-2) and multidrug resistant drug-associated proteins (MRPs), rendering cancer 

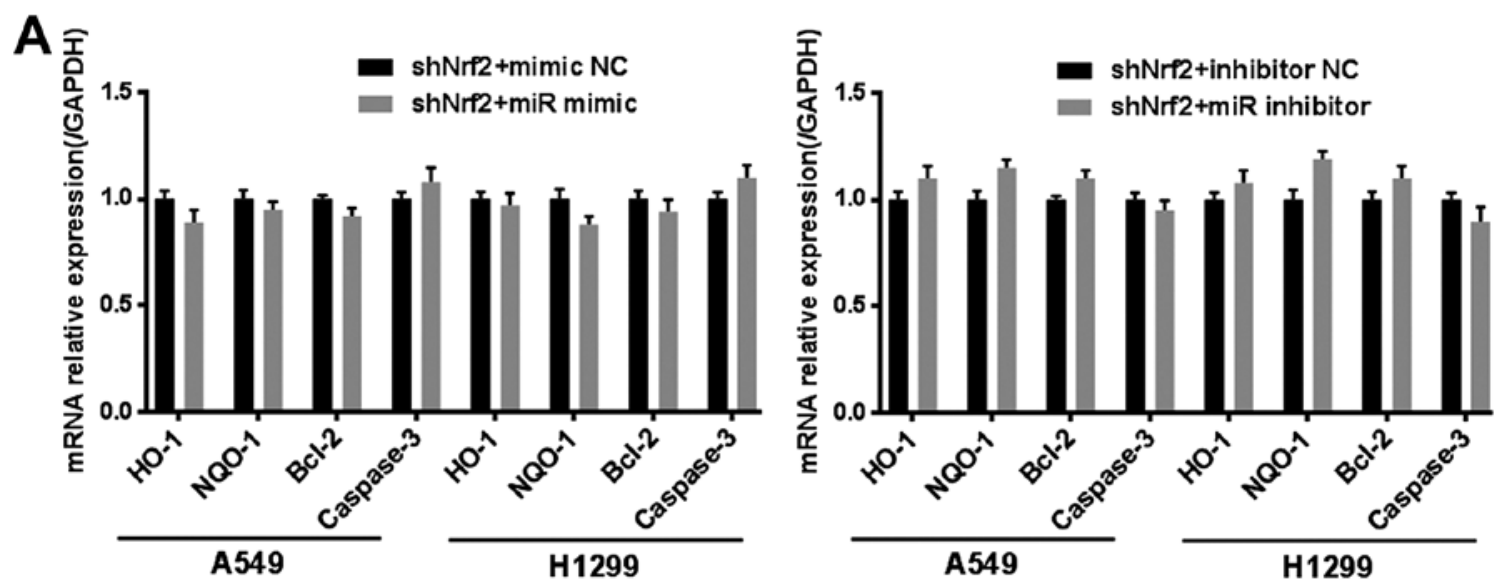

B

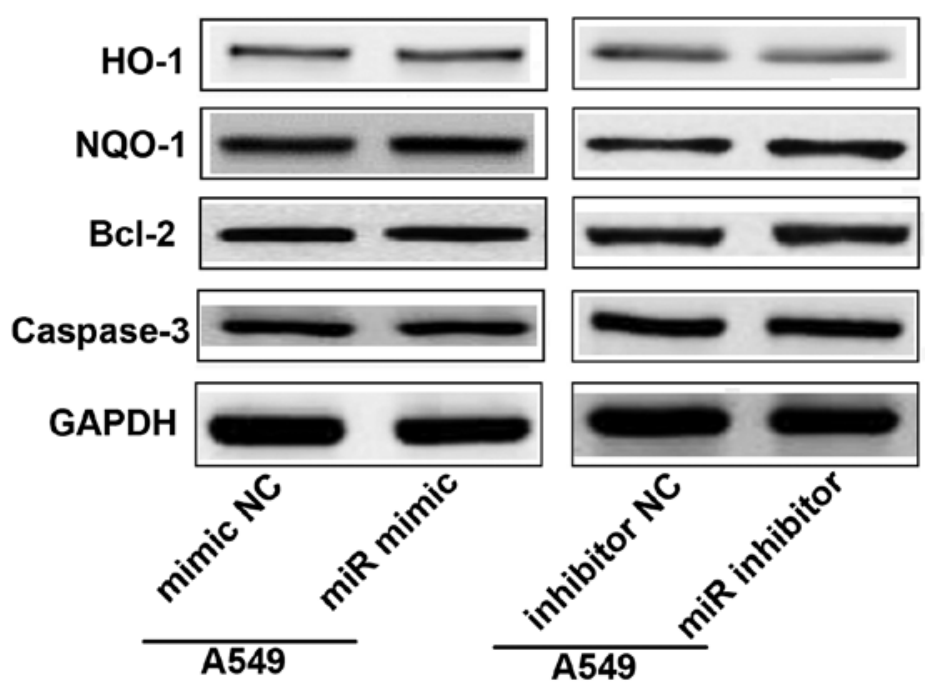

Figure 4. miR-144-3p promotes cisplatin sensitivity by adjusting Nrf2 in lung cancer cells. The confirmation that the role of miR-144-3p was achieved via Nrf2 was demonstrated by Real-time PCR and western blotting after knocking down endogenous Nrf2. The levels of these markers downstream were detected by (A) Real-time PCR and (B) western blotting $(n \geq 3)$.
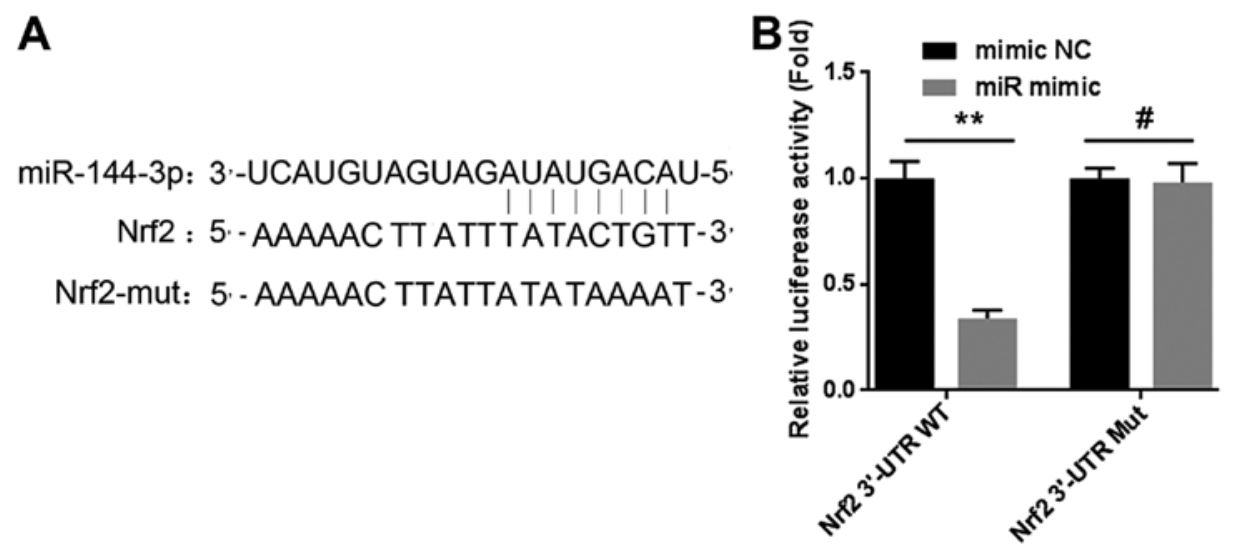

Figure 5. miR-144-3p mediates Nrf2 expression by targeting the 3'-UTR in lung cancer cells. (A) The predicted binding site of miR-144-3p in the 3'-UTR of Nrf2. (B) The relative luciferase activity of Cos-7 cells was detected after Nrf2-3'-UTR-WT or Nrf2-3'-UTR-MUT were co-transfected with miR-144-3p mimic and mimic $\mathrm{NC}\left(\mathrm{n} \geq 3 ;{ }^{* *} \mathrm{P}<0.01,{ }^{*} \mathrm{P}>0.05\right)$.

cells resistant to drugs (26-28). Nrf2 regulates downstream target gene expression mainly by binding with ARE elements in the target gene promoter region. When the activated Nrf2 enters the nucleus, and combines with the ARE sequence, the ARE-regulated genes involving metabolism, intracellular redox balance, apoptosis and drug resistance begin to transcribe and induce the expression of target genes and play the role of cell protection (29). Nrf2 has been revealed to be closely connected with cisplatin resistance $(30,31)$, however we know little about the relationship between miR-144-3p 

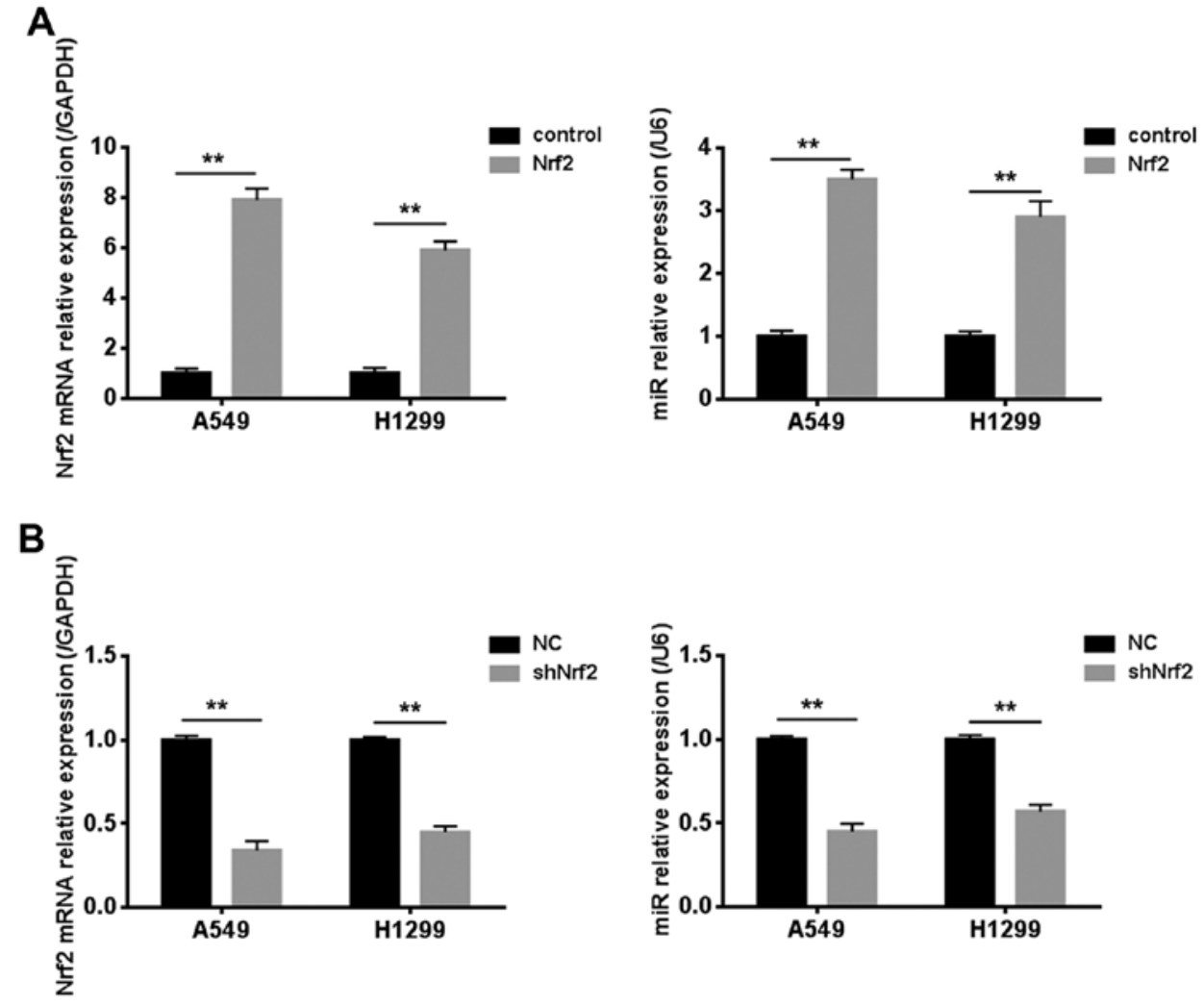

C
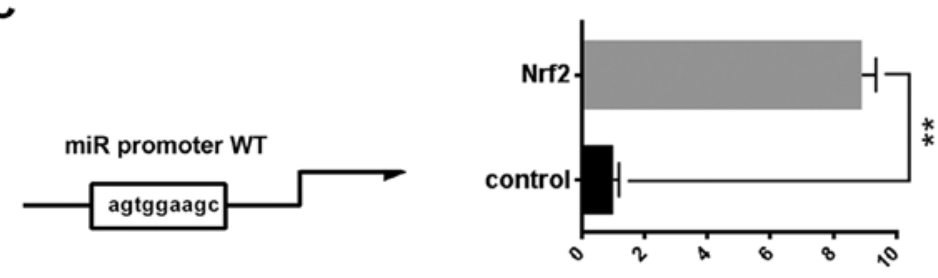

Relative luciferase activity (fold)
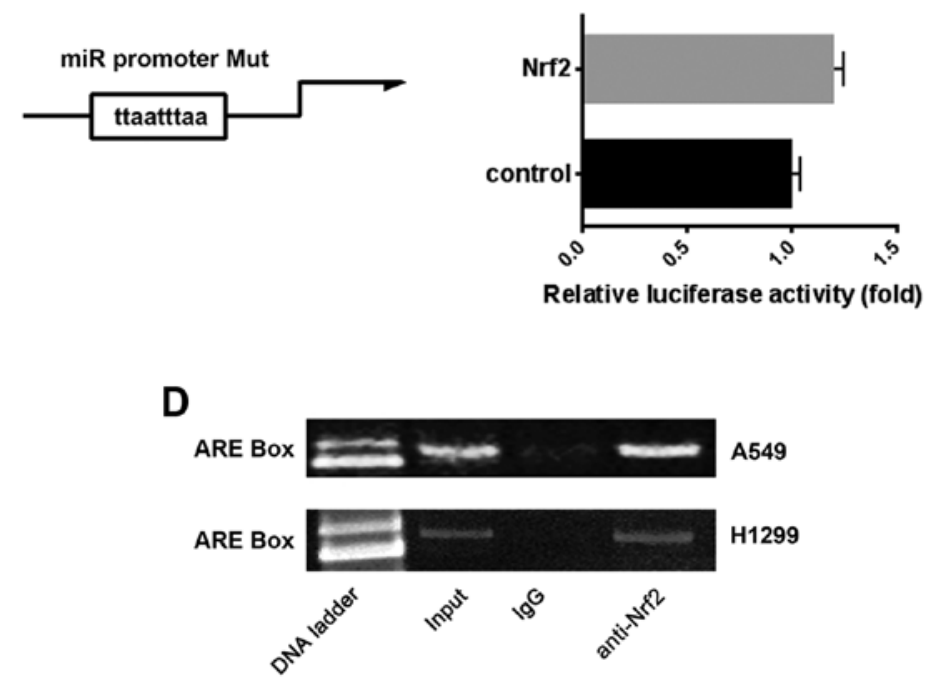

Figure 6. Nrf2 also affects the miR-144-3p expression by binding to the ARE box in the miR-144-3p promoter. (A and B) The expression of miR-144-3p was assessed by Real-time PCR and western blot assay after overexpression or knockdown of Nrf2 and normalized to GAPDH (n $\geq 3$; $\left.{ }^{* *} \mathrm{P}<0.01\right)$. (C) Cos-7 cells were transfected with Nrf2 and miR-144-3p-luc or mut-miR-144-3p-luc. A luciferase assay was performed $\left({ }^{* *} \mathrm{P}<0.01, \mathrm{n} \geq 3\right)$. The vector pcDNA3.1-was used as a negative control. (D) Chromatin immunoprecipitation (ChIP) assay was used to determine the sites on the miR-144-3p promoter binding with Nrf2 in A549 and H1299 cells.

and Nrf2. Our research findings revealed that targeting Nrf2 at a post-transcriptional stage via miR-144-3p could regulate its downregulation process, thus decreasing the expression of target genes related to drug resistance. 

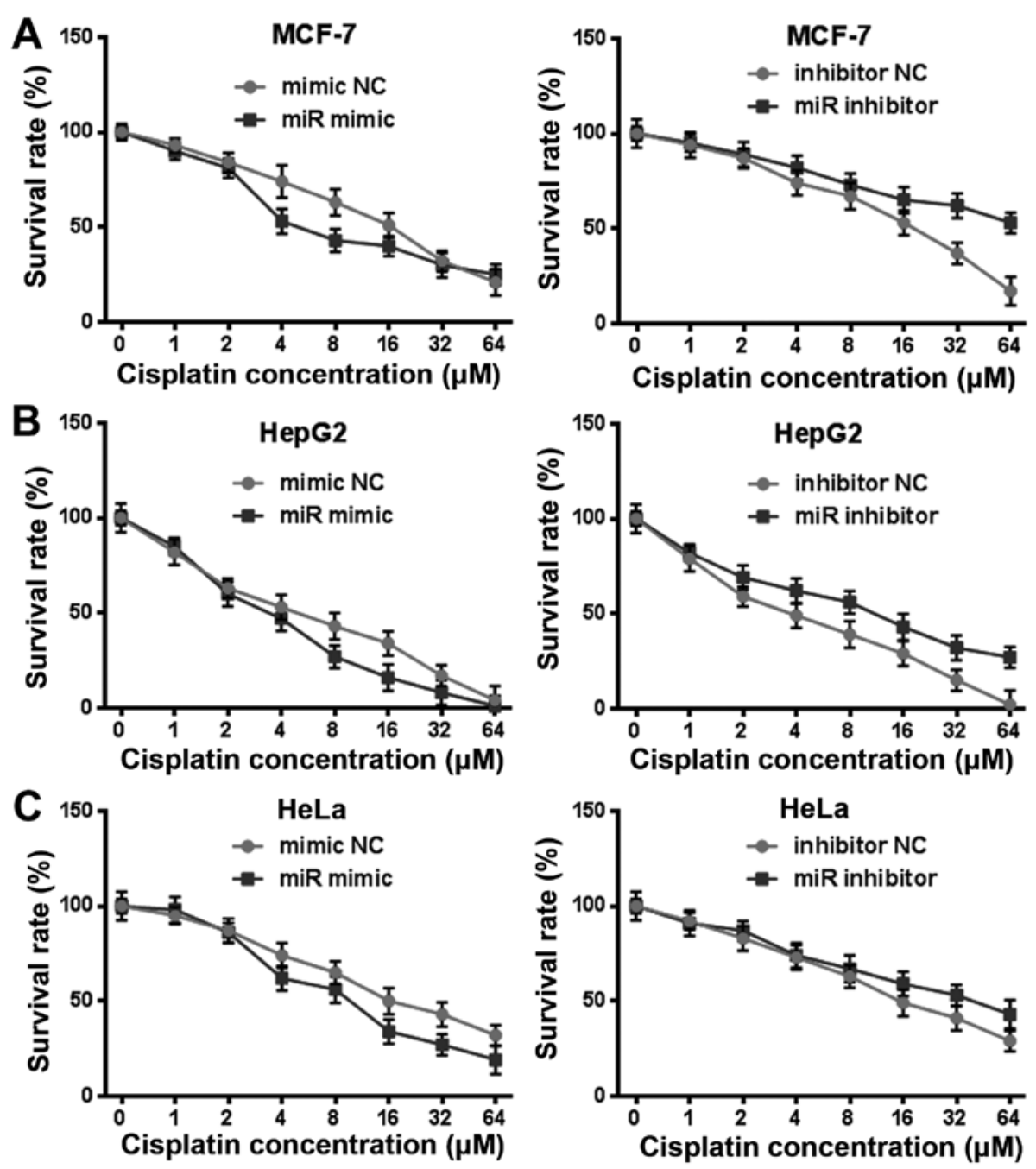

Figure 7. miR-144-3p also regulates cisplatin resistance in other tumor cells. Three types of tumor cells, overexpressing or with knocked down miR-144-3p, were treated with increasing levels of concentration $(1,2,4,8,16,32$ and $64 \mu \mathrm{M})$ of cisplatin for $24 \mathrm{~h}$. The survival rate of cells was determined using MTT assay. Data (mean $\pm \mathrm{SD}$ ) represent the mean value of three independent experiments.

In addition, we revealed that the overexpression of $\mathrm{Nrf} 2$ can activate the expression of miR-144-3p by binding the antioxidant response element (ARE) in the promoter region. Perhaps this is a self-protection mechanism of the body when drug resistance occurs during the chemotherapy procedure.

In conclusion, we determined that miR-144-3p expression was related with the survival rate and cisplatin resistance of lung cancer patients. Furthermore, there was a positive association between the levels of miR-144-3p and the sensitivity of lung cancer cells to cisplatin. Moreover, we demonstrated that $\mathrm{miR}-144-3 \mathrm{p}$ reacted to cisplatin resistance in lung cancer cells by altering its target Nrf2. Furthermore, overexpression of $\mathrm{Nrf} 2$, in turn, could regulate the expression of miR-144-3p by binding to ARE in the miR-144-3p promoter region. This may be a self-protection mechanism of the body. In addition, we also found that miR-144-3p could also regulate cisplatin resistance in other tumor cells. Cisplatin is an early-used and mature drug among antitumor drugs, but intrinsic and acquired resistance limits the clinical application of cisplatin. The present study, may provide some theoretical reference for the clinical inhibition of cisplatin resistance.

\section{Acknowledgements}

Not applicable.

\section{Funding}

The present study was financially supported by the Science and Technology Fund of Tianjin Municipal Health Bureau (2015KZ013).

\section{Availability of data and materials}

The datasets used and analyzed during the current study are available from the corresponding author on reasonable request.

\section{Author's contributions}

YY, HL and JQ designed the experiments. YY, HL, JX, DS, LZ, BL, LW and GL performed the experiments, analyzed and interpreted the data. YY and HL were major contributors in writing the manuscript. All authors read and approved the manuscript and agree to be accountable for all aspects of the 
research in ensuring that the accuracy or integrity of any part of the work are appropriately investigated and resolved.

\section{Ethics approval and consent to participate}

Not applicable.

\section{Patient consent for publication}

Not applicable.

\section{Competing interests}

The authors declare that they have no competing interests.

\section{References}

1. Jones GS and Baldwin DR: Recent advances in the management of lung cancer. Clin Med (Lond) 18 (Suppl 2): S41-S46, 2018

2. Chen W, Zheng R, Baade PD, Zhang S, Zeng H, Bray F, Jemal A, $\mathrm{Yu}$ XQ and He J: Cancer statistics in China, 2015. Cancer J Clin 66: 115-132, 2016.

3. Siegel RL, Miller KD and Jemal A: Cancer statistics, 2018. CA Cancer J Clin 68: 7-30, 2018.

4. Waqar SN and Morgensztern D: Treatment advances in small cell lung cancer (SCLC). Pharmacol Ther 180: 16-23, 2017.

5. Tsvetkova E and Goss GD: Drug resistance and its significance for treatment decisions in non-small cell lung cancer. Curr Oncol 19 (Suppl 1): S45-S51, 2012.

6. Barr MP, Gray SG, Hoffmann AC, Hilger RA, Thomale J, O'Flaherty JD, Fennell DA, Richard D, O'Leary JJ and O'Byrne KJ Generation and characterisation of cisplatin-resistant non-small cell lung cancer cell lines displaying a stem-like signature. PLoS One 8: e54193, 2013.

7. Dasari S and Tchounwou PB: Cisplatin in cancer therapy: Molecular mechanisms of action. Eur J Pharmacol 740: 364-378, 2014.

8. Rudolph C, Melau C, Nielsen JE, Vile Jensen K, Liu D, Pena-Diaz J, Rajpert-De Meyts E, Rasmussen LJ and Jørgensen A: Involvement of the DNA mismatch repair system in cisplatin sensitivity of testicular germ cell tumours. Cell Oncol (Dordr) 40: 341-355, 2017.

9. Chan M, Liaw CS, Ji SM, Tan HH, Wong CY, Thike AA, Tan PH, Ho GH and Lee AS: Identification of circulating microRNA signatures for breast cancer detection. Clin Cancer Res 19: 4477-4487, 2013.

10. Natalie G, Walker RC, Hee KC, Winter S and Hunter KW: Inherited variation in miR-290 expression suppresses breast cancer progression by targeting the metastasis susceptibility gene Arid4b. Cancer Res 73: 2671-2681, 2013.

11. Guarnieri DJ and Dileone RJ: MicroRNAs: A new class of gene regulators. Ann Med 40: 197-208, 2008

12. Wahid F, Shehzad AT, Khan T and Kim YY: MicroRNAs: Synthesis, mechanism, function, and recent clinical trials. Biochim Biophys Acta 1803: 1231-1243, 2010.

13. Hoelscher SC, Doppler SA, Dreßen M, Lahm H, Lange R and Krane M: MicroRNAs: Pleiotropic players in congenital heart disease and regeneration. J Thorac Dis 9 (Suppl 1): S64-S81, 2017.
14. Kastl L, Brown I and Schofield AC: miRNA-34a is associated with docetaxel resistance in human breast cancer cells. Breast Cancer Res Treat 131: 445-454, 2012.

15. Dongoran RA and Wu TY: Abstract 5269: Cryptotanshinone activate Nrf2 expression through microRNA regulations. Cancer Res 77 (Suppl 13): 5269, 2017.

16. Singh B, Ronghe AM, Chatterjee A, Bhat NK and Bhat HK: MicroRNA-93 regulates NRF2 expression and is associated with breast carcinogenesis. Carcinogenesis 34: 1165-1172, 2013.

17. Zhao L, Qi Y, Xu L, Tao X, Han X, Yin L and Peng J: MicroRNA-140-5p aggravates doxorubicin-induced cardiotoxicity by promoting myocardial oxidative stress via targeting Nrf2 and Sirt2. Redox Biol 15: 284-296, 2018.

18. Livak KJ and Schmittgen TD: Analysis of relative gene expression data using real-time quantitative PCR and the 2(-Delta Delta C(T)) method. Methods 25: 402-408, 2001.

19. Holohan C, Van Schaeybroeck S, Longley DB and Johnston PG: Cancer drug resistance: An evolving paradigm. Nat Rev Cancer 13: 714-726, 2013.

20. Housman G, Byler S, Heerboth S, Lapinska K, Longacre M, Snyder N and Sarkar S: Drug resistance in cancer: An overview. Cancers (Basel) 6: 1769-1792, 2014.

21. Periti P and Mini E: Drug resistance in cancer: An overview of the clinical aspects. J Chemother 1: 5-9, 1989

22. Jiao X, Zhao L, Ma M, Bai X, He M, Yan Y, Wang Y, Chen Q, Zhao X, Zhou M, et al: MiR-181a enhances drug sensitivity in mitoxantone-resistant breast cancer cells by targeting breast cancer resistance protein (BCRP/ABCG2). Breast Cancer Res Treat 139: 717-730, 2013.

23. Ma MT, He M, Wang Y, Jiao XY, Zhao L, Bai XF, Yu ZJ, Wu HZ, Sun ML, Song ZG and Wei MJ: miR-487a resensitizes mitoxantrone (MX)-resistant breast cancer cells (MCF-7/MX) to MX by targeting breast cancer resistance protein (BCRP/ABCG2). Cancer Lett 339: 107-115, 2013.

24. Meyer zu Schwabedissen HE and Kroemer HK: In vitro and in vivo evidence for the importance of breast cancer resistance protein transporters (BCRP/MXR/ABCP/ABCG2). Handb Exp Pharmacol: 325-371, 2011.

25. Zhang Q, Zhang B, Sun L, Yan Q, Zhang Y, Zhang Z, Su Y and Wang C: MicroRNA-130b targets PTEN to induce resistance to cisplatin in lung cancer cells by activating Wnt/ $\beta$-catenin pathway. Cell Biochem Funct 36: 194-202, 2018.

26. Niture SK and Jaiswal AK: Nrf2-induced antiapoptotic Bcl-xL protein enhances cell survival and drug resistance. Free Radic Biol Med 57: 119-131, 2013.

27. Niture SK and Jaiswal AK: Nrf2 protein up-regulates antiapoptotic protein $\mathrm{Bcl}-2$ and prevents cellular apoptosis. J Biol Chem 287: 9873-9886, 2012.

28. Young LC, Campling BG, Cole SP, Deeley RG and Gerlach JH: Multidrug resistance proteins MRP3, MRP1, and MRP2 in lung cancer: Correlation of protein levels with drug response and messenger RNA levels. Clin Cancer Res 7: 1798-1804, 2001.

29. Hayes JD, McMahon M, Chowdhry S and Dinkova-Kostova AT: Cancer chemoprevention mechanisms mediated through the Keap1-Nrf2 pathway. Antioxid Redox Signal 13: 1713-1748, 2010.

30. Solis LM, Behrens C, Dong W, Suraokar M, Ozburn NC, Moran CA, Corvalan AH, Biswal S, Swisher SG, Bekele BN, et al: Nrf2 and Keapl abnormalities in non-small cell lung carcinoma and association with clinicopathologic features. Clin Cancer Res 16: 3743-3753, 2010.

31. Homma S, Ishii Y, Morishima Y, Yamadori T, Matsuno Y, Haraguchi N, Kikuchi N, Satoh H, Sakamoto T, Hizawa N, et al: Nrf2 enhances cell proliferation and resistance to anticancer drugs in human lung cancer. Clin Cancer Res 15: 3423-3432, 2009. 